\title{
THERMAL PROBLEMS WITH RADIATION BOUNDARY CONDITIONS
}

\author{
By G. M. L. GLADWELL \\ (Solid Mechanics Division, Faculty of Engineering, University of Waterloo, \\ Waterloo, Ontario, Canada N2L 3G1)
}

J. R. BARBER

(Department of Mechanical Engineering and Applied Mechanics, University of Michigan, Ann Arbor, Michigan 48109, U.S.A.)

and Z. OLESIAK

(Institute of Mechanics, University of Warsaw, Warsaw, Poland)

[Received 16 June 1982]

\section{SUMMAR Y}

Contact or crack problems in thermoelasticity are usually analysed with the idealised boundary conditions of perfect conduction or perfect insulation. These boundary conditions, while simplifying the mathematics, sometimes lead to unrealistic, singular thermoelastic fields. This paper formulates axisymmetric static thermal problems for a half-space when one boundary condition corresponds to partial insulation, either inside or outside the circle $r=a, z=0$. Four important cases are considered and the problems are reduced to the solution of integro-differential equations of Abel type. In each case it is shown that the equation can be solved by using two simultaneous Fourier expansions of the unknown function.

\section{Introduction}

CONTACT and crack problems in static thermoelasticity are usually solved under the assumption that the temperature is prescribed on one part of the boundary and the heat flux on the remainder. This assumption is made to simplify the mathematics. When, for instance, the thermoelastic problem can be divided into a purely thermal problem and an elastic one (see, e.g., Olesiak and Sneddon (1), George and Sneddon (2)), then the thermal field can often be written down in explicit form without the necessity of solving an integral equation. However, there is an accompanying disadvantage in that the resulting thermoelastic field can have singular properties. For instance, in the simple problem of a uniform heat source $q_{0}$ applied over the portion $x^{2}+y^{2} \leqslant a^{2}$ of the otherwise insulated boundary $z=0$ of the halfspace $z \geqslant 0$, then (3) the normal thermoelastic displacement on the boundary outside the circle $r \leqslant a$ is proportional to $\ln (r / a)$, and so is infinite at infinity. This singular behaviour at infinity can be removed by changing the boundary condition outside the circle in such a way that the total heat flux 
entering the half-space is zero. It is shown that one way to do this is to replace the insulated condition by the more realistic radiation condition.

The physical aspects of this problem are the subject of the sequel. The purpose of the present paper is to formulate mixed boundary-value problems for the static temperature field under radiation conditions and to reduce them to integral equations that are suitable for computation.

In order to reduce the analytical complications, attention will be directed to axisymmetric problems for the half-space $z \geqslant 0$. The basic problem is to find the harmonic temperature field $T(r, z)$ in cylindrical polar coordinates $(\boldsymbol{r}, \boldsymbol{z})$, satisfying the conditions (Carslaw and Jaeger $(\mathbf{5})$ )

$$
\begin{array}{lll}
k_{1} \frac{\partial T}{\partial z}-h_{1} T=R_{1}(r), & r<a, & z=0, \\
k_{2} \frac{\partial T}{\partial z}-h_{2} T=R_{2}(r), & r>a, & z=0 .
\end{array}
$$

The functions $R_{1}(r), R_{2}(r)$ are supposed to be specified, but must be such that $T(r, z)$ is continuous, and $T(r, z) \rightarrow 0$ as $r^{2}+z^{2} \rightarrow \infty$.

Although it is possible to solve the mixed boundary-value problem as stated, it seemed to us to be useful to confine attention to the four limiting cases which appear in practice, in which one of the four quantities $h_{1} a, h_{2} a$, $k_{1}, k_{2}$ becomes so small compared to the other three that it may be neglected.

Before proceeding to the analysis, we may make two other simplifications. Introduce dimensionless coordinates $(\rho, \zeta)$ such that $r=a \rho, z=a \zeta$, and the dimensionless Biot numbers $h_{i} a / k_{t}=H_{t}, i=1,2$; then one limiting case of equations (1.1), (1.2) corresponding to $k_{2} \rightarrow 0$, is

$$
\begin{aligned}
& \frac{\partial T}{\partial \zeta}-H_{1} T=R(\rho), \quad \rho<1, \quad z=0, \\
& T=T_{2}(\rho), \quad \rho>1, \quad z=0,
\end{aligned}
$$

where $H_{1}=h_{1} a / k_{1}, R(\rho)=a R_{1}(r) / k_{1}$.

Let $S, S^{\prime}$ denote the regions $\rho<1$ and $\rho>1$ respectively on the surface of the half-space. The four basic physical problems are:

(i) $S$ is partially insulated while $S^{\prime}$ is completely conducting and held at constant temperature. On $\zeta=0$,

$$
\frac{\partial T}{\partial \zeta}-H_{1} T=R(\rho), \quad \rho<1 ; \quad T=0, \quad \rho>1 .
$$

(ii) $S$ is partially insulated while $S^{\prime}$ is completely insulated. On $\zeta=0$,

$$
\frac{\partial T}{\partial \zeta}-H_{1} T=R(\rho), \quad \rho<1 ; \quad \frac{\partial T}{\partial \zeta}=0, \quad \rho<1 .
$$


(iii) $S$ is kept at a specified temperature while $S^{\prime}$ is partially insulated. On $\zeta=0$,

$$
T=T_{0}, \quad \rho<1 ; \quad \frac{\partial T}{\partial \zeta}-H_{2} T=0, \quad \rho<1 .
$$

(iv) $S$ is subjected to a specified heat flux while $S^{\prime}$ is partially insulated. On $\zeta=0$,

$$
\frac{\partial T}{\partial \zeta}=F_{0}, \quad \rho<1 ; \quad \frac{\partial T}{\partial \zeta}-H_{2} T=0, \quad \rho>1 .
$$

In each case $\partial T / \partial \zeta=\partial T(\rho, 0) /\left.\partial \zeta\right|_{\zeta-0}, T \equiv T(\rho, 0)$ and $R(\rho)$ must satisfy regularity conditions so that $T(\rho, \zeta) \rightarrow 0$ as $\rho^{2}+\zeta^{2} \rightarrow \infty$.

It was noted in the introduction that the solutions of problems (iii) and (iv) have the important property that the total flux of heat into the half-space is zero, i.e.

$$
\int_{0}^{\infty} \rho \frac{\partial T}{\partial \zeta}(\rho, 0) d \rho=0 .
$$

This property is proved later.

\section{Preliminary analysis}

The analysis follows similar lines in each of the four cases. In each case the harmonic function $T(\rho, \zeta)$ is represented as a Hankel transform

$$
T(\rho, \zeta)=\mathscr{H}_{0}\left[\xi^{-1} A(\xi) \exp (-\xi \zeta) ; \rho\right],
$$

and extensive use is made of the relationships between Hankel, Fourier cosine, Fourier sine and Abel transforms. These are defined as follows (see (5) or (6), chapter 5):

Hankel: $\quad \mathscr{K}_{\nu}\left[\xi^{-1} f(\xi) ; \rho\right]=\int_{0}^{\infty} f(\xi) J_{\nu}(\xi \rho) d \xi ;$

Fourier: $\quad \mathscr{F}_{c}[f(\xi) ; x]=(2 / \pi)^{\frac{1}{1}} \int_{0}^{\infty} f(\xi) \cos (\xi x) d \xi$,

$$
F_{s}[f(\xi) ; x]=(2 / \pi)^{\frac{1}{2}} \int_{0}^{\infty} f(\xi) \sin (\xi x) d \xi
$$

Abel: $\quad \mathscr{A}_{1}[f(x) ; \rho] \quad=(2 / \pi)^{\frac{1}{2}} \int_{0}^{\rho}\left(\rho^{2}-x^{2}\right)^{-\frac{1}{1}} f(x) d x$,

$$
\mathscr{A}_{2}[f(x) ; \rho]=(2 / \pi)^{\frac{1}{2}} \int_{\rho}^{\infty}\left(x^{2}-\rho^{2}\right)^{-\frac{1}{2}} f(x) d x,
$$

with inverses

$$
\begin{aligned}
& \mathscr{A}_{1}^{-1}[F(\rho) ; x]=D_{x} \mathscr{A}_{1}[\rho F(\rho) ; x], \\
& \mathscr{A}_{2}^{-1}[F(\rho) ; x]=-D_{x} \mathscr{A}_{2}[\rho F(\rho) ; x],
\end{aligned}
$$

where $D_{x}=d / d x$ 
The following relationships are known (6, chapter 5):

$$
\begin{aligned}
\mathscr{A}_{1}^{-1}\left\{\mathscr{K}_{0}\left[\xi^{-1} A(\xi) ; \rho\right] ; x\right\} & =\mathscr{F}_{c}[A(\xi) ; x], \\
\mathscr{A}_{1}\left\{\rho \mathscr{K}_{0}[A(\xi) ; \rho] ; x\right\} & =\mathscr{F}_{s}[A(\xi) ; x], \\
\mathscr{A}_{2}^{-1}\left\{\mathscr{K}_{0}\left[\xi^{-1} A(\xi) ; \rho\right] ; x\right\} & =\mathscr{F}_{s}[A(\xi) ; x], \\
\mathscr{A}_{2}\left\{\rho \mathscr{K}_{0}[A(\xi) ; \rho] ; x\right\} & =\mathscr{F}_{c}[A(\xi) ; x] .
\end{aligned}
$$

In the solution of the integral equations extensive use is made of Legendre polynomials, and in particular the following results are used (see (7), 3.675):

$$
\begin{gathered}
\int_{\phi}^{\frac{1}{2} \pi} \frac{\sin (2 n+1) \theta d \theta}{\{2(\cos 2 \phi-\cos 2 \theta)\}^{\frac{1}{2}}}=\frac{1}{4} \pi P_{n}(\cos 2 \phi), \\
\int_{0}^{\phi} \frac{\cos (2 n+1) \theta d \theta}{\{2(\cos 2 \theta-\cos 2 \phi)\}^{\frac{1}{2}}}=\frac{1}{4} \pi P_{n}(\cos 2 \phi), \\
\frac{P_{n}(x)-P_{n+2}(x)}{1-x^{2}}=\frac{(2 n+3)}{2(n+1)(n+2)} \sum_{k=0}^{n}\left\{1+(-)^{n+k}\right\}(2 k+1) P_{k}(x) .
\end{gathered}
$$

\section{Case (i)}

The boundary conditions on $T(\rho, \zeta)$ are (1.5), namely

$$
\frac{\partial T}{\partial \zeta}-H T=R(\rho), \quad \rho<1 ; \quad T=0, \quad \rho>1,
$$

so that the Hankel form (2.1) yields the dual integral equations

$$
\begin{aligned}
\mathscr{K}_{0}[A(\xi) ; \rho]+H_{\mathscr{K}_{0}}\left[\xi^{-1} A(\xi) ; \rho\right] & =-R(\rho), & & \rho<1, \\
\mathscr{H}_{0}\left[\xi^{-1} A(\xi) ; \rho\right] & =0, & & \rho>1 .
\end{aligned}
$$

Apply $\mathscr{A}_{2}^{-1}$ to equation (3.3) and use (2.11) to obtain

$$
\mathscr{F}_{s}[A(\xi) ; x]=\left(\frac{1}{2} \pi\right)^{\frac{1}{2}} f(x) U(1-x),
$$

where $U$ is the Heaviside unit function. Now express equation (3.2) in terms of $f(x)$. Equations (2.10), (2.11) give

$$
\begin{aligned}
\mathscr{K}_{0}[A(\xi) ; \rho] & =\frac{1}{\rho} \mathscr{A}_{1}^{-1}\left\{\mathscr{F}_{s}[A(\xi) ; x] ; \rho\right\} \\
& =\frac{1}{\rho} \frac{d}{d \rho} \int_{0}^{\rho} \frac{x f(x) d x}{\left(\rho^{2}-x^{2}\right)^{\frac{1}{2}}}, \quad \rho<1,
\end{aligned}
$$

and

$$
\begin{aligned}
\mathscr{H}_{0}\left[\xi^{-1} A(\xi) ; \rho\right] & =\mathscr{A}_{2}\left\{F_{s}[A(\xi) ; x] ; \rho\right\} \\
& =\int_{\rho}^{1} \frac{f(x) d x}{\left(x^{2}-\rho^{2}\right)^{1}}, \quad \rho<1,
\end{aligned}
$$


so that

$$
\frac{1}{\rho} \frac{d}{d \rho} \int_{0}^{\rho} \frac{x f(x) d x}{\left(\rho^{2}-x^{2}\right)^{\frac{1}{2}}}+H \int_{\rho}^{1} \frac{f(x) d x}{\left(x^{2}-\rho^{2}\right)^{\frac{1}{2}}}=-R(\rho), \quad \rho<1 .
$$

In order to reduce this equation to a suitable form for computation we put $x=\cos \theta, \rho=\cos \phi$ and use two simultaneous representations for $f(x)$, namely

$$
\begin{aligned}
f(x)=F(\theta)=\sum_{n=0}^{\infty} a_{n} \cos (2 n+1) \theta, & 0<\theta<\frac{1}{2} \pi, \\
\sin \theta F(\theta)=\sum_{n=0}^{\infty} b_{n} \cos (2 n+1) \theta, & 0<\theta<\frac{1}{2} \pi,
\end{aligned}
$$

Then on using (2.13), (2.14) we find that

$$
\begin{aligned}
\int_{0}^{\rho} \frac{x f(x) d x}{\left(\rho^{2}-x^{2}\right)^{\frac{1}{2}}} & =\sum_{n=0}^{\infty} a_{n} \int_{\phi}^{\pi} \frac{\sin 2 \theta \cos (2 n+1) \theta d \theta}{\{2(\cos 2 \phi-\cos 2 \theta)\}^{\frac{1}{2}}} \\
& =\frac{\pi}{8} \sum_{n=0}^{\infty} a_{n}\left\{P_{n+1}(\cos 2 \phi)-P_{n-1}(\cos 2 \phi)\right\}
\end{aligned}
$$

where the ${ }^{*}$ indicates that the $P_{-1}$ occurring when $n=0$ is taken to be $P_{0}$. Similarly

$$
\begin{aligned}
\int_{\rho}^{1} \frac{f(x) d x}{\left(x^{2}-\rho^{2}\right)^{\frac{1}{2}}} & =2 \sum_{n=0}^{\infty} b_{n} \int_{0}^{\phi} \frac{\cos (2 n+1) \theta d \theta}{\{2(\cos 2 \phi-\cos 2 \theta)\}^{\frac{1}{2}}} \\
& =\frac{\pi}{2} \sum_{n=0}^{\infty} b_{n} P_{n}(\cos 2 \phi) .
\end{aligned}
$$

Now equation (2.15) and the rule for differentiation of a Legendre polynomial yield

$$
\frac{1}{\rho} \frac{d}{d \rho}\left\{P_{n+1}\left(2 \rho^{2}-1\right)-P_{n-1}\left(2 \rho^{2}-1\right)\right\}=4(2 n+1) P_{n}\left(2 \rho^{2}-1\right) .
$$

This holds also, when the above mentioned convention is used, for $n=0$, so that equation (3.7) becomes

$$
\frac{\pi}{2} \sum_{n=0}^{\infty}(2 n+1) a_{n} P_{n}\left(2 \rho^{2}-1\right)+\frac{\pi}{2} H \sum_{n=0}^{\infty} b_{n} P_{n}\left(2 \rho^{2}-1\right)=-R(\rho)
$$

Thus if we expand $R(\rho)$ in the form

$$
R(\rho)=\frac{\pi}{2} \sum_{n=0}^{\infty}(2 n+1) g_{n} P_{n}\left(2 \rho^{2}-1\right),
$$

then we have

$$
a_{n}+H(2 n+1)^{-1} b_{n}=-g_{n}, \quad n=0,1,2, \ldots
$$


This must be supplemented by the relationship between $a_{n}$ and $b_{n}$, namely

where

$$
b_{n}=\sum_{m=0}^{\infty} c_{m, n} a_{m}
$$

$$
\begin{aligned}
c_{m, n} & =\frac{2}{\pi} \int_{0}^{\pi} \sin \theta \cos (2 m+1) \theta \cos (2 n+1) \theta d \theta \\
& =\frac{1}{\pi}\left(\frac{1}{2 n+2 m+3}-\frac{1}{2 n+2 m+1}+\frac{1}{2 n-2 m+1}-\frac{1}{2 n-2 m-1}\right) .
\end{aligned}
$$

It may be shown that the set of equations (3.15), (3.16) is regular in the sense of Kantorovitch and Krylov (8); they may therefore be solved by truncation.

Collins (9) and Barber (10) reduced the problem (3.1) to the integral equation

$$
-\frac{H}{\pi} \int_{-1}^{1} \ln |t-x| f(t) d t+f(x)=-\frac{2}{\pi} \int_{0}^{x} \frac{\rho R(\rho) d \rho}{\left(x^{2}-\rho^{2}\right)^{\frac{1}{2}}} .
$$

This is seen to yield equations (3.15), (3.16) by noting that

$$
\begin{aligned}
-\frac{1}{\pi} \int_{-1}^{1} \ln |t-x| f(t) d t & =-\frac{1}{\pi} \int_{0}^{\pi} \ln |\cos \theta-\cos \phi| F(\theta) \sin \theta d \theta \\
& =-\frac{1}{\pi} \sum_{n=0}^{\infty} b_{n} \int_{0}^{\pi} \ln |\cos \theta-\cos \phi| \cos (2 n+1) \theta d \theta \\
& =\sum_{n=0}^{\infty}(2 n+1)^{-1} b_{n} \cos (2 n+1) \phi,
\end{aligned}
$$

and that equation (3.14) yields

$$
\frac{2}{\pi} \int_{0}^{x} \frac{\rho R(\rho) d \rho}{\left(x^{2}-\rho^{2}\right)^{\frac{1}{2}}}=\sum_{n=0}^{\infty} g_{n} \cos (2 n+1) \phi .
$$

\section{Case (ii)}

The boundary conditions are

$$
\frac{\partial T}{\partial \zeta}-H T=R(\rho), \quad \rho<1 ; \quad \frac{\partial T}{\partial \zeta}=0, \quad \rho>1,
$$

so that the Hankel forms yield the equations

$$
\begin{aligned}
\mathscr{K}_{0}[A(\xi) ; \rho]+H \mathscr{H}_{0}\left[\xi^{-1} A(\xi) ; \rho\right] & =-R(\rho), & & \rho<1, \\
\mathscr{H}_{0}[A(\xi) ; \rho] & =0, & & \rho>1 .
\end{aligned}
$$

Apply $\mathscr{A}_{2} \rho$ to (4.3) and use (2.13) to obtain

$$
\mathscr{F}_{\mathrm{c}}[A(\xi) ; x]=\left(\frac{1}{2} \pi\right)^{\frac{1}{t}} f(x) U(1-x),
$$


which, when substituted into (4.2), yields the Abel equation

$$
-\frac{1}{\rho} \frac{d}{d \rho} \int_{\rho}^{1} \frac{x f(x) d x}{\left(x^{2}-\rho^{2}\right)^{\frac{1}{2}}}+H \int_{0}^{\rho} \frac{f(x) d x}{\left(\rho^{2}-x^{2}\right)^{\frac{1}{2}}}=-R(\rho), \quad \rho<1 .
$$

The appropriate representations for $f(x)$ are now

for then

$$
\begin{aligned}
f(x) & =F(\theta)=\sum_{n=0}^{\infty} a_{n} \sin (2 n+1) \theta, \\
\sin \theta F(\theta) & =\sum_{n=0}^{\infty} b_{n} \sin (2 n+1) \theta,
\end{aligned}
$$

$$
\begin{aligned}
-\frac{1}{\rho} \frac{d}{d \rho} \int_{\rho}^{1} \frac{x f(x) d x}{\left(x^{2}-\rho^{2}\right)^{\frac{1}{2}}} & =\frac{\pi}{2} \sum_{n=0}^{\infty}(2 n+1) a_{n} P_{n}\left(2 \rho^{2}-1\right), \\
\int_{0}^{\rho} \frac{f(x) d x}{\left(\rho^{2}-x^{2}\right)^{\frac{1}{2}}} & =\frac{\pi}{2} \sum_{n=0}^{\infty} b_{n} P_{n}\left(2 \rho^{2}-1\right),
\end{aligned}
$$

and equation (4.5) again yields (3.15). The relationship between the $a_{n}$ and $b_{n}$ is now

where

$$
b_{n}=\sum_{m=0}^{\infty} d_{m, n} a_{m}
$$

$$
\begin{aligned}
d_{m, n} & =\frac{2}{\pi} \int_{0}^{\pi} \sin \theta \sin (2 n+1) \theta \sin (2 m+1) \theta d \theta \\
& =\frac{1}{\pi}\left(\frac{1}{2 n-2 m+1}-\frac{1}{2 n-2 m-1}-\frac{1}{2 n+2 m+3}+\frac{1}{2 n+2 m+1}\right) .
\end{aligned}
$$

We note that there are two other equivalent integral equations for $f(x)$, namely

$$
\int_{-1}^{1}\left\{\frac{1}{\pi(t-x)}+\frac{H}{2} \operatorname{sgn}(t-x)\right\} f(t) d t=\frac{2}{\pi} \int_{0}^{x} \frac{\rho R(\rho) d \rho}{\left(x^{2}-\rho^{2}\right)^{\frac{1}{2}}},
$$

and

$$
f(x)+\frac{H}{\pi} \int_{0}^{1} f(y) \ln \left|\frac{\left(1-x^{2}\right)^{\frac{1}{2}}+\left(1-y^{2}\right)^{\frac{1}{2}}}{\left(1-x^{2}\right)^{\frac{1}{2}}-\left(1-y^{2}\right)^{\frac{1}{2}}}\right| d y=-\frac{2}{\pi} \int_{x}^{1} \frac{\rho R(\rho) d \rho}{\left(\rho^{2}-x^{2}\right)^{\frac{1}{2}}} .
$$

Equation (4.12) appears in load-transfer problems and has been studied by Morar' and Popov (11), Arutiunian and Mkhitarian (12), and Erdogan and Gupta (13). Equations (4.13) may be solved by using the expansion

$$
\ln \left|\frac{\sin \theta+\sin \phi}{\sin \theta-\sin \phi}\right|=4 \sum_{m=0}^{\infty}(2 m+1)^{-1} \sin (2 m+1) \theta \sin (2 m+1) \phi
$$


With the representations (4.6), (4.7), both (4.12) and (4.13) yield equation (3.15), (4.11). It is shown in (6), chapter 7, that the set of equations (3.15), (4.11) is regular.

\section{Cose (iii)}

The boundary conditions on $T(\rho, \zeta)$ are

$$
T=1, \quad \rho<1 ; \quad \frac{\partial T}{\partial \zeta}-H T=0, \quad \rho>1 .
$$

Using the representation (2.1) we may, following Sneddon (14), reduce the problem to the solution of a Fredholm integral equation with non-singular kernel. However, the kernel involves the cosine-integral function and is not suitable for computational purposes. We therefore proceed as follows.

The field $T(\rho, \zeta)$ is continuous at $\rho=1, \zeta=0$, and satisfies the zero-flux condition (1.9). We write

$$
T=T_{1}+T_{2}+T_{3},
$$

where $T_{1}+T_{2}$ itself satisfies the continuity and zero-flux conditions. Choose $T_{1}, T_{2}$ so that

$$
\begin{array}{llll}
T_{1}=1, & \rho<1 ; & \frac{\partial T_{1}}{\partial \zeta}=0, & \rho>1, \\
T_{2}=0, & \rho<1 ; & T_{2}=F(\rho), & \rho>1 .
\end{array}
$$

The solution for $T_{1}$ is well known and, in particular,

$$
\frac{\partial T_{1}}{\partial \zeta}=-\frac{2}{\pi}\left(1-\rho^{2}\right)^{-1}, \quad \rho<1 ; \quad T_{1}=\frac{2}{\pi} \sin ^{-1}\left(\frac{1}{\rho}\right), \quad \rho>1 .
$$

For $F(\rho)$ we may take

$$
F(\rho)=-\frac{2}{\pi} \frac{\left(\rho^{2}-1\right)^{\frac{1}{2}}}{\rho^{2}}
$$

and then

$$
\frac{\partial T_{2}}{\partial \zeta}=\frac{2}{\pi \rho^{3}}\left\{\sin ^{-1} \rho-\rho\left(1-\rho^{2}\right)^{-\frac{1}{1}}\right\}, \quad \rho<1 ; \quad \frac{\partial T_{2}}{\partial \zeta}=\frac{1}{\rho^{3}}, \quad \rho>1 .
$$

The boundary conditions for $T_{3}$ are therefore

$$
\begin{gathered}
T_{3}=0, \quad \rho<1, \\
\frac{\partial T_{3}}{\partial \zeta}-H T_{3}=\frac{2 H}{\pi}\left\{\sin ^{-1}\left(\frac{1}{\rho}\right)-\frac{\left(\rho^{2}-1\right)^{\frac{1}{2}}}{\rho^{2}}\right\}-\frac{1}{\rho^{3}} \equiv R(\rho), \quad \rho>1 .
\end{gathered}
$$


Proceeding as before we use the Hankel solution to obtain

$$
\begin{array}{rlrl}
\mathscr{H}_{0}\left[\xi^{-1} A(\xi) ; \rho\right] & =0, & & \rho<1, \\
\mathscr{K}_{0}[A(\xi) ; \rho]+H \mathscr{K}_{0}\left[\xi^{-1} A(\xi) ; \rho\right] & =-R(\rho), & \rho>1 .
\end{array}
$$

Thus

$$
F_{c}[A(\xi) ; x]=\left(\frac{1}{2} \pi\right)^{\frac{1}{2}} f(x) U(x-1),
$$

and hence

$$
\frac{1}{\rho} \frac{d}{d \rho} \int_{\rho}^{\infty} \frac{x f(x) d x}{\left(x^{2}-\rho^{2}\right)^{\frac{1}{2}}}-H \int_{1}^{\rho} \frac{f(x) d x}{\left(\rho^{2}-x^{2}\right)^{\frac{1}{2}}}=R(\rho), \quad \rho>1 .
$$

Put

$$
x=1 / y, \quad \rho=1 / s, \quad f(x)=y^{2} j(y)
$$

then

$$
s^{2} \frac{d}{d s} \int_{0}^{s} \frac{s j(y) d y}{\left(s^{2}-y^{2}\right)^{\frac{1}{2}}}+H \int_{s}^{1} \frac{y j(y) d y}{\left(y^{2}-s^{2}\right)^{\frac{1}{2}}}=-S(s)
$$

where

$$
S(s)=\frac{1}{s} R\left(\frac{1}{s}\right)=\frac{2 H}{\pi}\left\{\frac{\sin ^{-1} s}{s}-\left(1-s^{2}\right)^{\frac{1}{2}}\right\}-s^{2} .
$$

In order to obtain appropriate representations for $j(y)$ we put $y=\cos \theta$, $s=\cos \phi$, and first take

$$
\begin{gathered}
j(y)=J(\theta)=\sum_{n=0}^{\infty} \alpha_{n} \cos 2 n \theta, \\
\sin \theta J(\theta)=\sum_{n=0}^{\infty} \beta_{n} \cos 2 n \theta,
\end{gathered}
$$

where the prime indicates that the first term is $\frac{1}{2} \beta_{0}$. Now

$$
\int_{0}^{s} \frac{j(y) d y}{\left(s^{2}-y^{2}\right)^{\frac{1}{2}}}=\frac{\pi}{2} \alpha_{0}+\frac{\pi}{4} \sum_{n=1}^{\infty} \alpha_{n}\left\{P_{n}\left(2 s^{2}-1\right)-P_{n-1}\left(2 s^{2}-1\right)\right\} .
$$

After some manipulation it is found that

$$
\frac{d}{d s}\left\{s\left(P_{n}-P_{n-1}\right)\right\}=(2 n+1) P_{n}+(2 n-1) P_{n-1}, \quad n \geqslant 1,
$$

where $P_{n} \equiv P_{n}\left(2 s^{2}-1\right)$ hereafter. Thus

$$
\begin{aligned}
\frac{d}{d s} \int_{0}^{s} \frac{s j(y) d y}{\left(s^{2}-y^{2}\right)^{\frac{1}{2}}} & =\frac{\pi}{2} \alpha_{0}+\frac{\pi}{4} \sum_{n=1}^{\infty} \alpha_{n}\left\{(2 n+1) P_{n}+(2 n-1) P_{n-1}\right\} \\
& =\frac{\pi}{4}\left(2 \alpha_{0}+\alpha_{1}\right)+\frac{\pi}{4} \sum_{n=1}^{\infty}(2 n+1)\left(\alpha_{n}+\alpha_{n+1}\right) P_{n} .
\end{aligned}
$$


In order to simplify this expression we note that if (5.17) is rewritten

then, since

$$
\cos \theta J(\theta)=\sum_{n=0}^{\infty} a_{n} \cos (2 n+1) \theta,
$$

$$
\frac{\cos (2 n+1) \theta}{\cos \theta}=2 \cos 2 n \theta-2 \cos (2 n-2) \theta+\ldots 2(-1)^{n-1} \cos 2 \theta+(-1)^{n},
$$

we have

$$
2 \alpha_{0}+\alpha_{1}=2 a_{0}, \quad \alpha_{n}+\alpha_{n+1}=2 a_{n}, \quad n \geqslant 1,
$$

so that

$$
\frac{d}{d s} \int_{0}^{s} \frac{s j(y) d y}{\left(s^{2}-y^{2}\right)^{\frac{1}{2}}}=\frac{\pi}{2} \sum_{n=0}^{\infty}(2 n+1) a_{n} P_{n} .
$$

The second integral in (5.15) is

$$
\int_{s}^{1} \frac{y j(y) d y}{\left(s^{2}-y^{2}\right)^{\frac{1}{2}}}=\frac{\pi}{4} \beta_{0}+\frac{\pi}{4} \sum_{n=0}^{\infty} \beta_{n+1}\left(P_{n+1}+P_{n}\right)
$$

The sum on the right is divisible by $s^{2}$; in fact

$$
\frac{P_{n+1}+P_{n}}{s^{2}}=\frac{2}{n+1}\left\{(2 n+1) P_{n}-(2 n-1) P_{n-1}+\ldots+(-1)^{n} P_{0}\right\}
$$

so that $Q_{n} \equiv\left(P_{n}+P_{n-1}\right) / 2 s^{2}$ satisfies the equations

$$
Q_{1}=1, \quad n Q_{n}+(n+1) Q_{n+1}=(2 n+1) P_{n} .
$$

Thus, since the first term in (5.15) and $S(s)$ on the right are both divisible by $s^{2}$, we deduce that $H \beta_{0}=0$. But

$$
H \beta_{0}=\frac{2 H}{\pi} \int_{0}^{\pi} \sin \theta J(\theta) d \theta=\frac{2 H}{\pi} \int_{0}^{1} j(y) d y=\frac{2 H}{\pi} \int_{1}^{\infty} f(x) d x,
$$

so that if $H \neq 0$, then

$$
\int_{1}^{\infty} f(x) d x=0 .
$$

This is the zero-flux condition mentioned earlier.

In order to simplify (5.26) we use (5.28) which shows that if (5.18) is rewritten

$$
\sin \theta J(\theta)=\sum_{n=0}^{\infty}(2 n+1)^{-1} b_{n}\{n \cos 2 n \theta+(n+1) \cos (2 n+2) \theta\},
$$

where

$$
\frac{n+1}{2 n+1} b_{n}+\frac{n+1}{2 n+3} b_{n+1}=\beta_{n+1}, \quad n=0,1, \ldots
$$


then

$$
\frac{1}{s^{2}} \int_{s}^{1} \frac{y j(y) d y}{\left(s^{2}-y^{2}\right)^{1}}=\frac{\pi}{2} \sum_{n=0}^{\infty} b_{n} P_{n} .
$$

If therefore we may expand $S(s) / s^{2}$ in the form

$$
\frac{S(s)}{s^{2}}=\frac{\pi}{2} \sum_{n=0}^{\infty}(2 n+1) g_{n} P_{n}
$$

then equation (5.15) reduces to equation (3.15)!

In order to expand $S(s)$ in terms of the $P_{n}$ we note that

$$
\begin{aligned}
\frac{\sin ^{-1} s}{s} & =\frac{\pi}{2 s}-\frac{1}{s}\left(\frac{\pi}{2}-\sin ^{-1} s\right)=\int_{s}^{1} \frac{d \rho}{\rho\left(1-\rho^{2}\right)^{\frac{1}{2}}\left(\rho^{2}-s^{2}\right)^{\frac{1}{2}}}-\int_{s}^{1} \frac{d \rho}{\rho\left(\rho^{2}-s^{2}\right)^{\frac{1}{2}}} \\
& =2 \int_{0}^{\phi} \frac{1-\sin \theta}{\cos \theta} \frac{d \theta}{\{2(\cos 2 \theta-\cos 2 \phi)\}^{\frac{1}{2}}}, \text { with } s=\cos \phi .
\end{aligned}
$$

Suppose then that

then

$$
\frac{1-\sin \theta}{\cos \theta}=\sum_{m=0}^{\infty} c_{m} \cos (2 m+1) \theta
$$

$$
\frac{\sin ^{-1} s}{s}=\frac{\pi}{2} \sum_{m=0}^{\infty} c_{m} P_{m}\left(2 s^{2}-1\right)
$$

The coefficients $c_{m}$ are found to be given by

$$
\frac{\pi}{4} c_{m}=(-1)^{m}\left\{\frac{\pi}{2}-2\left(1-\frac{1}{3}+\frac{1}{5}-\ldots+\frac{(-1)^{m}}{2 m+1}\right)+\frac{(-1)^{m}}{2 m+1}\right\},
$$

and we note that $c_{m} \sim(2 m+1)^{-1}$ for large $m$. In a similar way we note that

Thus if

$$
\left(1-s^{2}\right)^{\frac{1}{1}}=\int_{s}^{1} \frac{\rho d \rho}{\left(\rho^{2}-s^{2}\right)^{\frac{1}{2}}}=\int_{0}^{\phi} \frac{2 \sin \theta \cos \theta d \theta}{\{2(\cos 2 \theta-\cos 2 \phi)\}^{\frac{1}{2}}} .
$$

then

$$
\frac{1}{2} \sin 2 \theta=\sum_{m=0}^{\infty} d_{m} \cos (2 m+1) \theta
$$

$$
\left(1-s^{2}\right)^{\frac{1}{2}}=\frac{\pi}{2} \sum_{m=0}^{\infty} d_{m} P_{m}\left(2 s^{2}-1\right)
$$

The coefficients $d_{m}$ are given by

$$
\frac{\pi}{4} d_{m}=\frac{1}{2} \int_{0}^{\frac{1}{2} \pi} \sin 2 \theta \cos (2 m+1) \theta d \theta=-\frac{1}{(2 m+3)(2 m-1)}
$$

Thus

$$
S(s)=H \sum_{m=0}^{\infty} e_{m} P_{m}-s^{2}, \quad e_{m}=c_{m}-d_{m} .
$$


Since $S(s) / s^{2}$ is finite as $s \rightarrow 0$, we may rewrite (5.42) in the form

$$
S(s)=H \sum_{m=0}^{\infty} f_{m}\left(P_{m}+P_{m+1}\right)-s^{2},
$$

where

$$
f_{m}=e_{m}-e_{m-1}+\ldots+(-1)^{m} e_{0} \quad \text { and } \quad f_{m} \rightarrow 0 \quad \text { as } m \rightarrow \infty .
$$

Then by using (5.27) we find that the coefficients $g_{n}$ in (5.32) are given by

$$
\mathrm{g}_{n}=\frac{4 H}{\pi} \sum_{m=0}^{\infty} \frac{(-1)^{m} f_{m+n}}{m+n+1}-\frac{2}{\pi} \delta_{n 0} .
$$

The equations (3.15) must be supplemented by the relation between the $a_{n}$ and $b_{n}$. These may be found in three stages. The $a_{n}$ are related to the $\alpha_{n}$ by (5.24), the $b_{n}$ to the $\beta_{n}$ by (5.30), and the $\alpha_{n}$ to the $\beta_{n}$ by

$$
\beta_{n}=\sum_{m=0}^{\infty} e_{m, n} \alpha_{m},
$$

where

$$
\begin{aligned}
e_{m, n} & =\frac{2}{\pi} \int_{0}^{\pi} \sin \theta \cos 2 m \theta \cos 2 n \theta d \theta \\
& =\frac{1}{\pi}\left(\frac{1}{2 m+2 n+1}-\frac{1}{2 m+2 n-1}+\frac{1}{2 m-2 n+1}-\frac{1}{2 m-2 n-1}\right) .
\end{aligned}
$$

\section{Case (iv)}

The boundary conditions on $T$ are

$$
\frac{\partial T}{\partial \zeta}=1, \quad \rho<1 ; \quad \frac{\partial T}{\partial \zeta}-H T=0, \quad \rho>1 .
$$

We divide $T$ into two parts:

$$
T=T_{1}+T_{2},
$$

where

$$
\frac{\partial T_{1}}{\partial \zeta}=1, \quad \rho<1 ; \quad T_{1}=0, \quad \rho>1 .
$$

The solution for $T_{1}$ is well known and, in particular

$$
\left.\begin{array}{c}
T_{1}(\rho, 0)=-\frac{2}{\rho}\left(1-\rho^{2}\right)^{\frac{1}{2}}, \quad \rho<1 ; \\
\frac{\partial T_{1}}{\partial \zeta}(\rho, 0)=\frac{2}{\pi}\left\{\sin ^{-1}\left(\frac{1}{\rho}\right)-\left(\rho^{2}-1\right)^{-\frac{1}{2}}\right\}, \quad \rho>1 .
\end{array}\right\}
$$


Thus the boundary conditions for $T_{2}$ are

$$
\begin{gathered}
\frac{\partial T_{2}}{\partial \zeta}=0, \quad \rho<1, \\
\frac{\partial T_{2}}{\partial \zeta}-H T_{2}=-\frac{2}{\pi}\left\{\sin ^{-1}\left(\frac{1}{\rho}\right)-\left(\rho^{2}-1\right)^{-\frac{1}{2}}\right\} \equiv R(\rho), \quad \rho>1 .
\end{gathered}
$$

Again, since $T_{1}$ is continuous at $\rho=1, z=0$, and satisfies the zero-flux condition, $T_{2}$ will satisfy these conditions also.

Using the Hankel form (2.1) we find

$$
\begin{aligned}
& \mathscr{H}_{0}[A(\xi) ; \rho]=0, \quad \rho<1, \\
& \mathscr{H}_{0}[A(\xi) ; \rho]+H \mathscr{H}_{0}\left[\xi^{-1} A(\xi) ; \rho\right]=-R(\rho), \quad \rho>1,
\end{aligned}
$$

so that

$$
\begin{gathered}
F_{s}[A(\xi) ; x]=\left(\frac{1}{2} \pi\right)^{\frac{1}{2} f} f(x) U(x-1), \\
\frac{1}{\rho} \frac{d}{d \rho} \int_{1}^{\rho} \frac{x f(x) d x}{\left(\rho^{2}-x^{2}\right)^{\frac{1}{2}}}+H \int_{\rho}^{\infty} \frac{f(x) d x}{\left(x^{2}-\rho^{2}\right)^{\frac{1}{2}}}=-R(\rho), \quad \rho>1 .
\end{gathered}
$$

Using the substitutions (5.14) we find

where

$$
-s^{2} \frac{d}{d s} s \int_{s}^{1} \frac{j(y) d y}{\left(y^{2}-s^{2}\right)^{\frac{1}{2}}}+H \int_{0}^{s} \frac{y j(y) d y}{\left(s^{2}-y^{2}\right)^{\frac{1}{2}}}=-S(s),
$$

$$
S(s)=\frac{1}{s} R\left(\frac{1}{s}\right)=-\frac{2}{\pi}\left\{\frac{\sin ^{-1} s}{s}-\left(1-s^{2}\right)^{-\frac{1}{2}}\right\} .
$$

Put $y=\cos \theta, s=\cos \phi$ and use the representations

$$
\begin{aligned}
& j(y)=J(\theta)=\sum_{n=1}^{\infty} a_{n} \sin 2 n \theta, \\
& \sin \theta J(\theta)=\sum_{n=1}^{\infty} \beta_{n} \sin 2 n \theta .
\end{aligned}
$$

The representation (6.14) automatically satisfies the zero-flux conditions. Then

$$
\begin{aligned}
\int_{s}^{1} \frac{j(y) d y}{\left(y^{2}-s^{2}\right)^{\frac{1}{2}}} & =\sum_{n=1}^{\infty} \alpha_{n} \int_{0}^{\phi} \frac{2 \sin \theta \sin 2 n \theta d \theta}{\{2(\cos 2 \theta-\cos 2 \phi)\}^{\frac{1}{2}}} \\
& =\frac{\pi}{4} \sum_{n=1}^{\infty} \alpha_{n}\left\{P_{n-1}-P_{n}\right\},
\end{aligned}
$$

so that, on using (5.20), we find that

$$
\begin{aligned}
U_{1} & \equiv-\frac{d}{d s} s \int_{s}^{1} \frac{j(y) d y}{\left(y^{2}-s^{2}\right)^{\frac{1}{2}}}=\frac{\pi}{4} \sum_{n=1}^{\infty} \alpha_{n}\left\{(2 n-1) P_{n-1}+(2 n+1) P_{n}\right\} \\
& =\frac{\pi}{4} \alpha_{1}+\frac{\pi}{4} \sum_{n=1}^{\infty}(2 n+1)\left(\alpha_{n}+\alpha_{n+1}\right) P_{n} .
\end{aligned}
$$


Proceeding as in section 5 we note that

$$
\frac{\sin (2 n+1) \theta}{\cos \theta}=2 \sin 2 n \theta-2 \sin (2 n-2) \theta+\ldots+2(-1)^{n-1} \sin 2 \theta+(-1)^{n} \frac{\sin \theta}{\cos \theta},
$$

so that if $J(\theta)$ is written in the form

where $\cos \theta J(\theta)=\sum_{n=0}^{\infty} a_{n} \sin (2 n+1) \theta$,

$$
\sum_{n=0}^{\infty}(-1)^{n} a_{n}=0
$$

then

so that

$$
\begin{gathered}
\alpha_{1}=2 a_{0}, \quad \alpha_{n}+\alpha_{n+1}=2 a_{n}, \\
U_{1}=\frac{\pi}{2} \sum_{n=0}^{\infty}(2 n+1) a_{n} P_{n} .
\end{gathered}
$$

The second integral can be treated similarly:

$$
V_{1} \equiv \int_{0}^{3} \frac{y j(y) d y}{\left(s^{2}-y^{2}\right)^{\frac{1}{2}}}=\frac{\pi}{4} \sum_{n=1}^{\infty} \beta_{n}\left\{P_{n}+P_{n-1}\right\}
$$

so that if (6.14) is rewritten as

$$
\sin \theta J(\theta)=\sum_{n=0}^{\infty}(2 n+1)^{-1} b_{n}\{n \sin 2 n \theta+(n+1) \sin (2 n+2) \theta\},
$$

where the $b_{n}$ and $\beta_{n}$ are related by equation (5.31), then

$$
\frac{1}{s^{2}} V_{1}=\frac{\pi}{2} \sum_{n=0}^{\infty} b_{n} P_{n} \text {. }
$$

If therefore we expand $S(s) / s^{2}$ in the form (5.33) then equation (6.11) also reduces to equation (3.15).

The two remaining matters are the representation of $S(s)$ and the relation between the $a_{n}$ and $b_{n}$. The first term in $S(s)$ is given in (5.36) and for the second we note that $\left(1-s^{2}\right)^{-\frac{1}{2}}$ has the formal expansion

$$
\left(1-s^{2}\right)^{-1}=\frac{\pi}{2} \sum_{m=0}^{\infty} e_{m} P_{m}, \quad e_{m}=4 / \pi .
$$

Now the $g_{n}$ are found as in section 5. The $a_{n}, \alpha_{n}$ are related by (6.19), (6.20), the $b_{n}, \beta_{n}$ by (5.31), and the $\alpha_{n}, \beta_{n}$ by

$\begin{aligned} f_{m, n} & =\frac{2}{\pi} \int_{0}^{\pi} \sin \theta \sin 2 m \theta \sin 2 n \theta d \theta \\ = & \frac{1}{\pi}\left(\frac{1}{2 m+2 n-1}-\frac{1}{2 m+2 n+1}+\frac{1}{2 m-2 n+1}-\frac{1}{2 m-2 n-1}\right) .\end{aligned}$ 
Finally, we note that the problem (6.6) may also be reduced to the Fredholm integral equation

$$
f(x)+\frac{H}{\pi} \int_{1}^{\infty} f(t) \ln \left|\frac{\left(x^{2}-1\right)^{\frac{1}{2}}+\left(t^{2}-1\right)^{\frac{1}{4}}}{\left(x^{2}-1\right)^{\frac{1}{2}}-\left(t^{2}-1\right)^{\frac{1}{2}}}\right| d t=-\frac{2}{\pi} \int_{1}^{x} \frac{\rho R(\rho) d \rho}{\left(x^{2}-\rho^{2}\right)^{\frac{1}{2}}}, \quad x>1 .
$$

This equation may be solved by approximation using (5.14), (6.13) and (6.14) or (6.18), (6.23).

\section{Conchusions}

Each of the four basic mixed boundary-value problems relating to the radiation condition has been reduced to an Abel type of integro-differential equation. In each case the use of appropriate Fourier representations reduces the problem basically to the same infinite set of simultaneous linear equations.

In cases (i) and (ii), it is known that the equations are regular. In cases (iii) and (iv), the regularity is not easily proved, but numerical experiments showed that the approximate solutions obtained by truncation exhibit good behaviour. Problems (ii) and (iii) are discussed further in the sequel.

\section{Ackmowledgements}

This work started when two of the authors (J.R.B. and Z.O.) were visiting the third at the University of Waterloo under a grant from the National Science and Engineering Research Council of Canada.

\section{REFERENCES}

1. Z. Olesiak and I. N. Sneddon, Arch. ration. Mech. Anal. 4 (1960) 238.

4. D. L. George and I. N. SNedDON, J. Math. Mech. 11 (1962) 665.

3. J. R. BARBer, Int. J. engng Sci. 9 (1971) 1165.

4. H. S. Carslaw and J. C. Jaeger, Conduction of Heat in Solids (Clarendon Press, Oxford, 1959).

5. I. N. SNeddon, Fourier Transforms (McGraw-Hill, New York, 1951).

6. G. M. L. Gladwell, Contact Problems in the Classical Theory of Elasticity (Sijthoff and Noordhoff, Alphen aan den Rijn, 1980).

7. I. S. GradshtEYN and I. M. RYzhik, Tables of Integrals, Series and Products (Academic Press, New York and London, 1965).

8. L. V. Kantorovich and V. I. KRYLOV, Approximate Methods of Higher Analysis, English translation A. D. Benster, (Interscience, New York, 1958).

9. W. D. Collins, Mathematika 6 (1959) 120.

10. J. R. Barber, $Q$. $J$ Mech. appl. Math. 29 (1976) 1.

11. G. A. Morar' and G. LA. Popov, J. appl. Math. Mech. 34 (1971) 389.

12. G. A. Arutiunian and S. M. Mkhttarian, in R. E. Czarnota-Bojarski et al. (editors), Trends in Elasticity and Thermoelasticity, Witold Nowacki Anniversary Volume (Wolters Noordhoff, Gröningen, 1971).

13. F. ERdogAN and G. D. GuPTA, J. appl. Mech. 38 (1971) 937.

14. I. N. SNEDdon, Mixed Boundary Value Problems in Potential Theory (North Holland, Amsterdam, 1966) 106. 